\section{RevistadePolírica Económica y Desarrollo Sostenible}

Centro Internacional de Política Económica para el Desarrollo Sostenible
Revista de Política Económica y Desarrollo Sostenible

EISSN: 2215-4167 • Vol. 3 (2) • Enero-Junio, 2018: 1-5

DOI: http://dx.doi.org/10.15359/peds.3-2.3

URL: http://www.revistas.una.ac.cr/politicaeconomica

Revista electrónica semestral publicada por el Centro Internacional de Política Económica para el Desarrollo Sostenible Universidad Nacional, Lagunilla, Heredia Apartado 2393-3000

\title{
Ideas Latinoamericanas sobre el Enfoque de Cadenas Globales de Mercancías
}

\author{
Álvaro Martín Parada Gómez \\ (iD https://orcid.org/0000-0002-8876-0869
}

Autor contacto:agomez@una.cr

\begin{abstract}
Resumen
El análisis de cadenas globales de valor (CGV) como marco teórico y metodológico ha sido muy utilizado en la academia (universidades), en el gobierno para la toma de decisiones sectoriales (ministerios) y en la elaboración de estrategias para el mejoramiento de la competitividad (empresas). Desde esta perspectiva, el libro Ideas latinoamericanas sobre el enfoque de Cadenas Globales de Mercancías, el cual se comentará en este editorial, plantea problemáticas centradas en múltiples factores críticos que afectan a sectores productivos: la calidad, el financiamiento, el comercio internacional, los mercados imperfectos, entre otros. El libro fue publicado por la Editorial de la Universidad Nacional Autónoma de México y editado por Rafael Díaz Porras, Wim Peluppesy y Pablo Pérez Akaki.
\end{abstract}

\section{Introducción}

A través del libro, los investigadores han hecho un esfuerzo científico significativo en explicar a profundidad las cuatro dimensiones de las CGV: 1. La dimensión geográfica, 2. El insumo-producto; 3. la fuerza motriz y; 4. La dimensión institucional. Las aplicaciones de las cadenas han sido en países diferentes como Colombia, México, Costa Rica, entre otros. Esto ha permitido comprender realidades distintas que mejorar las posturas teóricas desde el punto de vista epistemológico. Los resultados obtenidos por cada investigador en cada una de las temáticas abordadas permiten comprender el enfoque de cadenas globales de valor adecuadamente. Las contribuciones de la publicación son desarrolladas de la siguiente forma: la coordinación en las cadenas globales de valor; el poder de mercado y la fuerza motriz en las cadenas globales de valor; las posibilidades de análisis estratégico en las cadenas globales de valor; los mercados se dinamizan no solo por precios en las cadenas globales de valor, los cuáles serán comentados a continuación.

\section{Desarrollo}

\section{a) La coordinación en las cadenas globales de valor}

El enfoque de cadenas ha planteado una base teórica, estableciendo cadenas orientadas por la oferta y aquellas orientadas por la demanda. Adicional, se ha extendido formulando

1 Académico Escuela de Economía, Universidad Nacional, Costa Rica. Posee un Doctorado en Economía y Desarrollo por la Universidad de Tilburg, Holanda. 
teóricamente cadenas con coordinación jerárquica de tipo vertical, especializada, dependiente y débil coordinación (esta adición ha sido bien desarrollada en estudios empíricos propuestos como la cadena del sector turismo (Ochoa 2015, p.87), el sector cafetero (Pérez y Velázquez, 2011, p. 23).

En el enfoque teórico se establece un marco de trabajo estructurado que orienta el análisis de un producto o servicio generado por las empresas en distintos sectores productivos. Esto es posible porque la cadena es una amplia red de trabajo establecida por eslabones que transitan desde la apropiación de materias primas hasta el consumo final o desecho. La relación entre los eslabones podría ser a través de una integración vertical u horizontal en la empresa. En este caso se inicia con la explicación del eslabón de apropiación de insumos o materias primas, seguido por el análisis del diseño, pasando al proceso de producción. El cuarto eslabón explica las condiciones de mercadeo y comercialización, finalizando con el consumo final. Al respecto integra muy bien el concepto de estructura y proceso en el desarrollo productivo. Muchos autores del libro, hacen contribuciones a través de la investigación en productos particulares como: café (Pérez y Velázquez 2015, p 23). Este enfoque es integrador comparativamente con enfoques de orden más cuantitativo como lo son los típicos modelos econométricos que asumen causalidades teóricamente determinadas y que simplifican la realidad significativamente. Entre las razones se puede indicar que el análisis de la dimensión institucional en las cadenas asume la existencia de fallas de mercado, por lo tanto, debe haber mecanismos de intervención en el sistema económico. En términos generales, el análisis del desarrollo productivo a través de las cadenas exige entender la relación entre el mercado y la intervención del Estado dentro de una realidad compleja imposible de explicar modelísticamente.

\section{b) El poder de mercado y la fuerza motriz en las cadenas globales de valor}

Los mercados perfectos son más la excepción que la norma en la dinámica de producción global. El principio de competencia perfecta afirma que los precios son iguales a los ingresos marginales y costos marginales, la variable determinante son los precios, los cuales asignan los recursos productivos en el sistema económico. La contribución de los autores consiste en que, las cadenas globales de valor han evidenciado, en su mayoría, mercados imperfectos causados por economías de escala, integración vertical, externalidades, información imperfecta. Esta identificación es producto del análisis de la dimensión de la fuerza motriz al analizar la producción de café y el sector turismo (Díaz, 2015; Ochoa, 2015). De igual forma el pensamiento neoclásico indica que estas imperfecciones han conducido a concentración de mercado o poder de mercado (Bain, 1951). La fuerza motriz es analizada como una dimensión de la cadena, definiendo la estructura de control explicando la apropiación de beneficios a través de la concentración de la actividad económica, perpetuándose estructuras organizativas de mercados de carácter monopólico y oligopólico. Los procesos de integración vertical muestran como las corporaciones transnacionales se han percatado de ejercer poder sobre el consumidor al entrar en diferentes segmentos de forma extensiva en la cadena (Ochoa 2015, p. 91). El mismo autor afirma que el poder jerárquico de las empresas logra ejercer dominio sobre la demanda, controlando efectivamente el 
Revista de Política Económica • EISSN: 2215-4167

Vol. 3 (2) • Julio-Diciembre, 2018: 1-6

DOI: http://dx.doi.org/10.15359/peds.3-2.3

Parada Gómez

URL: http://www.revistas.una.ac.cr/politicaeconomica

mercado. Las características de la fuerza motriz analizadas por Ochoa (2015) y Díaz (2015) indican que las empresas definen la organización y control de la cadena a través de la determinación de la producción y comercialización en los productores, superan de forma explicativa en mucho la propuesta neoclásica que indica la valoración de concentración a través del Índice de concentración de Herfindahl e Hirschman (IHH) que integra variables específicas como el precio y el costo marginal. Por lo contrario, la fuerza motriz integra el análisis del poder de mercado de forma más amplia en cuanto a criterios distintos con múltiples variables en el análisis de los mercados (Díaz y Pelupessy, 2015; Jiménez y Pelupessy, 2015). Por lo tanto, la medición es relevante pero no determinante en el análisis del poder de los mercados. Por ejemplo, la presencia de diferenciación de productos, la innovación, la intensidad de la publicidad, conducen a distorsionar el mercado porque modifican el patrón de comportamiento de los consumidores.

La base teórica de la cadena supera en mucho el planteamiento neoclásico tradicional del mercado, el cual señala que las fuerzas ejercidas entre demandantes y oferentes a través del mecanismo de precios con llevan al bienestar. La superación radica en la integración de múltiples variables que explican las relaciones económicas, sociales, ambientales y culturales necesarias en la producción de un bien y servicio. Esta integración no puede ser explicada únicamente a través de los precios. Por lo tanto, el análisis de cadena ha logrado superar el enfoque de mercado integrando variables no precios como la calidad, la diferenciación de productos y la innovación. Al respecto, distintos autores concuerdan en el análisis de cuatro dimensiones en las cadenas, tales como: la dimensión geográfica, la estructura insumo producto, la fuerza motriz y la dimensión institucional, lo cual orienta metodológicamente el análisis de cadenas (Díaz,2015 ; Ochoa, 2015; Pérez 2015,). El integrar estas dimensiones supone la necesidad de analizar la función del mercado y el Estado en la organización de la estructura productiva.

\section{c) Las posibilidades de análisis estratégico en las cadenas globales de valor}

La incidencia de las investigaciones en cadenas globales de valor se puede ubicar en dos ámbitos. El primero, centrado en las estrategias de mejoramiento que trascienden desde el diseño, el proceso productivo, el mercadeo y la comercialización en la dinámica interna de las empresas que son parte de las cadenas. Adicionalmente, ha sido posible vincular desde la teoría neoclásica enfoques como el de teoría de juegos al análisis estratégico de las empresas en los mercados. Al respecto, Jiménez (2011), al analizar productos agroalimenticios como la producción de alverja China, indica que la teoría de juegos aporta al análisis de las cadenas globales de valor porque permiten comprender el nivel estratégico de competición en los mercados oligopólicos. Esto quiere decir que el enfoque de cadenas puede ser complementado con otros métodos de análisis, en este caso se integra la perspectiva microeconómica desde la teoría neoclásica. Por ejemplo, se explica la relación entre productores y procesadores de alverja China en Guatemala a través de la elaboración de un juego vertical estratégico considerando la participación de los agentes o actores sociales, el tipo de tecnología utilizada, el acceso a los mercados y controles de calidad ambiental establecidos 
en los acuerdos comerciales (Jiménez 2012, p. 92). También, el autor afirma que el enfoque de cadenas permite analizar el comportamiento estratégico de los agentes ubicados tanto en el mismo segmento de la cadena, como en diferentes segmentos de poder (Jiménez, 2015, p.153). Esto lo confirma con la experiencia hecho por Purwaningrum en la cadena de algodón en Uzbekistan. Con seguridad la teoría de juegos contribuye con sus métodos de análisis en la explicación de la concentración de poder inducida por estrategias de actores sociales que participan en segmentos distintos de la cadena. Esto pone en evidencia, lo relevante de la conducta de los productores y consumidores para explicar la apropiación de beneficios en los mercados.

El segundo es la incidencia de las cadenas en el diseño de la política pública relacionada con la estructura productiva. Al respecto, los investigadores coinciden en que las cadenas globales de valor proporcionan un marco de trabajo funcional para aproximarse a una política productiva adecuada. Al respecto se ha sugerido: el fortalecimiento del valor agregado en los productos y servicios, el impulso a la innovación tecnológica (Jiménez 2015, p.166), generación de escalamiento en la agricultura e industria mediante la ampliación de la escala en la empresa y redes de producción (Basualto, 2015, p.68); el fortalecimiento a la calidad de los productos acorde a las preferencias de los consumidores para posicionarse mejor en los mercados domésticos e internacionales a través del cumplimiento de certificaciones internacionales(Jiménez 2015, p.164; Pérez y Velázquez, 2015, p.26;). Posiblemente, en los múltiples análisis realizados en el ámbito de cadenas se identifican políticas productivas de apoyo a las empresas que las integran.

\section{d) Los mercados se dinamizan no solo por precios en las cadenas globales de valor}

Los precios es la variable estratégica en la asignación de los recursos en los mercados. No obstante, desde el enfoque de cadenas globales de valor se ha logrado integrar variables que modifican el patrón de comportamiento de los agentes económicos en la asignación de los recursos. Por ejemplo, en el caso de la producción de café de Costa Rica, Díaz ha evidenciado que los mercados son distorsionados por factores no precio tales como la calidad, diferenciación e innovación; por ejemplo, la integración de la innovación en productos y procesos productivos (Díaz, 2003, p.119). Desde mi punto de vista, estos factores trascienden al modelo tradicional de mercado que identifica la cantidad demanda en función de los precios (ley de demanda) y orienta a la vez las cantidades ofrecidas. Desde el enfoque de cadena global de valor, el factor de la calidad del producto es el resultado de una serie de procesos de mejoramiento en el diseño, las materias prima, la producción y la comercialización. Este hecho significa que el consumidor quiebra su patrón de comportamiento en el mercado orientando sus decisiones en favor de la calidad y no respondiendo a los estímulos de los bajos precios de forma estricta. Entonces, el criterio de competición no son los precios sino la calidad y la diferenciación como atributo creado por la empresa (ventaja competitiva) (Porter, 2011, p.104). En países en desarrollo como los de América Latina es común que los consumidores sean seguidores de los bajos precios porque los ingresos familiares son bajos en su mayoría y no permiten discriminar con base al criterio de calidad. Al respecto, Porter (1990) afirma que el nivel de ingreso medio y alto incentiva la 
exigencia de la calidad en los productos ofertados por las empresas, de ahí lo relevante de mejorar los ingresos de las economías de ingresos bajos y medios. Metodológicamente, Porter integra este criterio en la construcción del diamante de la competitividad al referirse a las condiciones de la demanda. No obstante, esto no significa que estratos de ingresos medios y altos no respondan al estímulo del vector de precios a la baja en el consumo de los bienes en los distintos mercados.

\section{Conclusión}

Las ideas latinoamericanas sobre el enfoque de Cadenas Globales de Mercancías (CGM) son el bastión de análisis teórico y metodológico de los sectores productivos para incidir en la toma de decisiones en el ámbito público y privado en países en desarrollo. Cada investigador y autor de cada temática que compone el libro muestra un análisis riguroso de las dimensiones de las CGM en su forma teórica y metodológica, relacionando la teoría y práctica en el ámbito de cada realidad referenciada.

El enfoque de CGM es integrador comparativamente con enfoques teóricos de orden más cuantitativos como los modelos econométricos. También, se hacen cuestionamientos al funcionamiento de los mercados de productos, explicándose la fuerza motriz como dimensión de análisis que detalla las fallas de mercado, así como otros factores socio económicos que influyen en la estructura de poder en las cadenas.

El debate de ideas en las CGM trasciende el análisis de los variables precios en los mercados y avanza hacia la integración de variables no precios como lo son: la innovación, la calidad, la diferenciación de productos, entre otras. Incluso, el debate se amplia hacia factores culturales, ambientales y geográficos que sin duda alguna tienen explicabilidad sobre problemas como la generación de valor agregado, la competitividad de las empresas, el bienestar socio económico, entre otras.

\section{Referencias}

Basulto, A (2015). El escalamiento en la industria electrónica en el norte de México. En Rafael Díaz, Wim Pelupessy y Pablo Pérez A (Ed). Ideas latinoamericanas sobre el enfoque de cadenas globales de mercancías. Universidad Nacional Autónoma de México. Facultad de Estudios Superiores Acatlan: Estado de México.

Díaz, R. (2003). A developing country perspective on policies for sustainable agribusiness chains: The case of Costa Rica. Ph.D. Thesis. Development Research Institute. Tilburg University: The Nederland.

Díaz, R. y Pelupessy W (2015). El impacto del control global al desarrollo local. En Rafael Díaz, Wim Pelupessy y Pablo Pérez A (Ed). Ideas latinoamericanas sobre el enfoque de cadenas 
globales de mercancías. Universidad Nacional Autónoma de México. Facultad de Estudios Superiores Acatlan: Estado de México.

Jiménez, G (2011). Sustainable Upgrading of Smallholders in Global Agri-food Chains. Ph.D. Thesis. Development Research Institute. Tilburg University: The Nederland.

Jiménez, G. y Pelupessy, W (2015). Estrategias de mejorar el ambiente en cadenas agroalimentarias. En Rafael Díaz, Wim Pelupessy y Pablo Pérez A (Ed). Ideas latinoamericanas sobre el enfoque de cadenas globales de mercancías. Universidad Nacional Autónoma de México. Facultad de Estudios Superiores Acatlan: Estado de México.

Ochoa, G (2015). El control y la coordinación transnacional y la política en el turismo de la Amazonia colombiana. En Rafael Díaz, Wim Pelupessy y Pablo Pérez A (Ed). Ideas latinoamericanas sobre el enfoque de cadenas globales de mercancías. Universidad Nacional Autónoma de México. Facultad de Estudios Superiores Acatlan: Estado de México.

Pérez, P y Velázquez, M. (2015). El mando en las cadenas de valor del café de sistemas tradicionales y alternativos en México. En Rafael Díaz, Wim Pelupessy y Pablo Pérez A (Ed). Ideas latinoamericanas sobre el enfoque de cadenas globales de mercancías. Universidad Nacional Autónoma de México. Facultad de Estudios Superiores Acatlan: Estado de México.

Porter, M (2011). ¿Qué es la estrategia?. Harvard Business Review. 89 (11); 100-117. https://dialnet. unirioja.es/servlet/articulo?codigo $=3802858$ 